\title{
The Revised Korean Practice Parameter for the Treatment of Attention-Deficit Hyperactivity Disorder (IV)
}

\author{
- Non-Pharmacologic Treatment -
}

\author{
Yun Mi Shin ${ }^{1}$, Eui-Jung Kim², Yunsin $\mathrm{Kim}^{3}$, Soo Young Bhang ${ }^{4}$, Eunha Lee ${ }^{5}$, \\ Cheol-Soon Lee ${ }^{6}$, Hyoung Yoon Chang ${ }^{1}$, Minha Hong ${ }^{7}$, and Dongwon Shin ${ }^{3}$ \\ ${ }^{1}$ Department of Psychiatry, Ajou University School of Medicine, Suwon, Korea \\ ${ }^{2}$ Department of Psychiatry, School of Medicine, Ewha Womans University, Seoul, Korea \\ ${ }^{3}$ Department of Psychiatry, Sungkyunkwan University School of Medicine, Kangbuk Samsung Hospital, Seoul, Korea \\ ${ }^{4}$ Department of Psychiatry, Eulji University School of Medicine, Eulji University Eulji Hospital, Seoul, Korea \\ ${ }^{5}$ The ADD Centre and Biofeedback Institute of Toronto, Toronto, Canada \\ ${ }^{6}$ Department of Psychiatry, Gyeongsang National University School of Medicine, Gyeongsang National University Changwon Hospital, \\ Changwon, Korea \\ ${ }^{7}$ Department of Psychiatry, Seonam University School of Medicine, Myongji Hospital, Goyang, Korea
}

\section{주의력결핍 과잉행동장애 한국형 치료 권고안(IV) \\ - 비약물적 치료 -}

신윤미 ${ }^{1}$, 김의정 ${ }^{2}$, 김윤신 ${ }^{3}$, 방수영 $^{4}$, 이은하 ${ }^{5}$, 이철순 $^{6}$, 장형윤 $^{1}$, 홍민하 $^{7}$, 신동원 $^{3}$

아주대학교 의과대학 정신건강의학교실, ${ }^{\prime}$ 이화여자대학교 의학전문대학원 정신건강의학교실, ${ }^{2}$

성균관대학교 의과대학 강북삼성병원 정신건강의학교실, ${ }^{3}$ 을지대학교 의과대학 을지대학교 을지병원 정신건강의학교실, ${ }^{4}$

$\mathrm{ADD}$ 센터, 토론토 바이오피드백 연구소, ${ }^{5}$ 경상대학교 의과대학 창원경상대학교병원 정신건강의학교실,

서남대학교 의과대학 명지병원 정신건강의학교실군

\begin{abstract}
Attention-deficit hyperactivity disorder (ADHD) is a neuropsychiatric disorder that begins in early childhood and can persist throughout adulthood. ADHD causes difficulties in various area of life, such as academic achievement, peer relationships, family functioning, employment and marriage. Although ADHD is known to respond well to medication, such treatment is more effective when combined with psychosocial (non-pharmacologic) therapy in terms of alleviating the core symptoms and improving appropriate functions. Psychosocial treatment interventions are divided into psychoeducation, behavioral parent training, school intervention, cognitive behavior therapy, social skill training, parent-child interaction therapy, play therapy, other treatments (coaching, complementary and alternative medicine), neurofeedback and Cogmed. Adult ADHD cognitive behavioral therapy is described separately. These practice parameters summarize the evidence for psychosocial treatment. Based on this evidence, specific recommendations are provided for psychosocial interventions.
\end{abstract}

Key Words: Attention-deficit hyperactivity disorder; Non-pharmacologic treatment; Practice parameter.

Received: February 24, 2017 / Revision: March 11, 2017 / Accepted: March 11, 2017

Address for correspondence: Dongwon Shin, Department of Psychiatry, Sungkyunkwan University School of Medicine, Kangbuk Samsung Hospital, 29 Saemunan-ro, Jongno-gu, Seoul 03181, Korea

Tel: +82-2-2001-2213, Fax: +82-2-2001-2211, E-mail: ntour@unitel.co.kr

\section{비약물적 치료}

Attention-deficit hyperactivity disorder(ADHD)는 초기 아동기부터 시작되어 성인기까지 생애 전반에 걸쳐 지속되는

This is an Open Access article distributed under the terms of the Creative Commons Attribution Non-Commercial License (http://creativecommons.org/licenses/by-nc/4.0) which permits unrestricted non-commercial use, distribution, and reproduction in any medium, provided the original work is properly cited.
신경정신의학적 장애이며 삶의 다양한 영역(학업, 또래관계, 가족 관계, 취업, 결혼, 건강, 금전관리, 운전 등)에서 심각한 어 려움과 손상을 겪게 된다. $\mathrm{ADHD}$ 는 약물치료에 대한 반응이 좋은 질환으로 알려져 있지만 핵심 증상을 호전시키고, 적절 한 기능을 발휘하기 위해서는 약물치료와 심리사회적(비약물 적) 치료가 병합되었을 때 효과적인 것으로 알려져 있다. 심리 사회적 치료적 개입은 발달단계에 따라 다양하다. 심리사회적 
치료적 개입을 정신의학적 교육, 행동적 부모훈련(behavioral parent training, BPT), 특수교육적 개입(행동 개입, 학습 개입, 자기 조절력 개입, 사회적 효능감 향상 전략), 인지행동치료, 사 회성훈련프로그램, 부모-아동 상호작용치료, 놀이치료, 기타치 료[코칭(coaching)], 보완대체의학(complementary and alternative medicine), 뉴로피드백, 코그메드(cogmed 작업기억력 훈련) 등으로 나누어 설명하고자 한다. 성인기 $\mathrm{ADHD}$ 환자 인 지행동치료는 따로 기술하였다.

\section{정신의학적 교육(Psychoeducation)}

$\mathrm{ADHD}$ 환자와 가족에게 $\mathrm{ADHD}$ 의 증상, 경과, 만성화 과정, 치료법과 이용 가능한 서비스 등에 대해 교육하는 것은 장기 적인 치료 경과에 있어서 치료에 대한 순응도를 높여줄 수 있 다는 점에서 중요하다. $\mathrm{ADHD}$ 의 여러 진료지침에서는 이러한 정신의학적 교육이 $\mathrm{ADHD}$ 의 치료 경과에 일정 부분 도움을 줄 수 있다고 권고하고 있다. ${ }^{1,2)}$ 특히 영국 국립보건임상연구소 (National Institute for Health and Care Excellence) 진료지 침에서는 학령전기 아동에서 부모훈련과 더불어 부모에게 정 신의학적 교육을 하는 것을 일차 치료로 권고하고 있다. 또한 $\mathrm{ADHD}$ 증상이 중등도 정도인 학령기 아동에서도 부모를 위한 정신의학적 교육을 하는 것의 중요성을 강조하고 있다. 정신의 학적 교육이 $\mathrm{ADHD}$ 의 핵심 증상을 경감시키는지에 대해 여전 히 논란이 되고 있기는 하나, 최근에는 여러 무작위대조군 임 상 연구(randomized controlled trial, RCT)에서 정신의학적 교 육이 부주의, 과잉행동, 충동성 등 $\mathrm{ADHD}$ 의 핵심 증상을 경 감시킨다는 결과들을 보고하고 있다. ${ }^{3-5)}$ 또한 정신의학적 교육 은 $\mathrm{ADHD}$ 의 핵심 증상 경감 효과뿐 아니라 환자의 행동, 환 자 및 부모의 만족도를 개선시키고 나아가 치료에 대한 순응 도를 향상시킨다는 보고가 있다. ${ }^{\circ}$ 또한 최근에는 정신의학적 교육의 장기적인 효과에 대한 연구도 진행된 바가 있는데 1년 이 지난 시점에서도 사회성 개선 효과가 지속된다고 보고하였 다.) 환자와 가족에 대한 정신의학적 교육은 다음의 몇 가지에 대해 중점적으로 시행하는 것이 도움이 된다. ${ }^{7)}$

1) $\mathrm{ADHD}$ 에 대한 기존의 지식에 대해 물어보고 잘못된 지 식이나 선입견이 있는지 파악한다.

2) 현재 사용하고 있는 $\mathrm{ADHD}$ 의 정확한 진단명을 알려주고 필요한 경우에 유사한 여러 명칭에 대해 혼동하지 않도록 설 명해준다.

3) $\mathrm{ADHD}$ 의 원인은 환경에서 기인하는 것이 아니며 신경생 물학적인 질환임을 설명해준다.

4) $\mathrm{ADHD}$ 는 다양한 증상을 나타낼 수 있지만, 어떤 환자들 은 일부의 증상만 보이기도 한다는 점을 설명해준다. 같은 진 단을 가진 타 환자들의 증상과 다른 양상을 보일 때 혼돈스
러워 하지 않도록 돕는다.

5) 부주의, 과잉행동, 충동성 등의 세 가지가 3대 핵심 증상 임을 설명해줌으로써 치료의 목표 증상에 집중할 수 있도록 해준다.

6) 3대 증상 외에도 기타 다양한 증상이 나타날 수 있고 생 활에 지장을 줄 수 있다는 것을 설명해준다.

7) 증상들은 때때로 정상적인 행동이 과잉으로 표출되는 경우임을 설명해준다. 따라서 이러한 경우에는 증상을 없애는 것이 아니라 적절한 수준으로 조절해야 하는 것임을 알게 해 준다.

8) 증상은 환자 자신이나 부모의 잘못 때문에 생겨난 것이 아님을 분명히 해주고, 치료 과정을 통해 개선될 수 있음을 알 려준다. 이로써 불필요한 원망을 막고 치료받는 것을 섣불리 포기하지 않도록 격려해준다.

9) $\mathrm{ADHD}$ 는 장기간 지속될 수 있으며 나이가 들어감에 따라 증상이 변화할 수 있음을 설명해줌으로써 장기간의 치료 가능 성에 대해 준비할 수 있도록 해준다.

10) 치료를 포기하거나 방관하지 않도록 격려해준다. 치료 를 받지 않는 경우에 발생할 수 있는 이차적인 문제들에 대해 설명해준다.

11) $\mathrm{ADHD}$ 치료가 $\mathrm{ADHD}$ 의 2차적 문제들을 예방할 수 있 음을 설명해준다.

12) 약물치료, 비약물적 치료법 등 다양한 치료법에 대해 소 개해주고 각각의 장단점 및 검증된 결과에 대해 설명해준다.

$\mathrm{ADHD}$ 에서 정신의학적 교육에 대한 연구들은 아동청소년 환자들의 부모를 대상으로 시행한 것이 대부분인데 최근에는 성인 $\mathrm{ADHD}$ 환자들에 대한 연구들이 보고되고 있다. 성인 $\mathrm{ADHD}$ 환자에서 정신의학적 교육과 인지행동치료 간 RCT 비교 연구 결과를 보면, $\mathrm{ADHD}$ 핵심 증상 및 자존감 개선, 우울 및 불안 증상의 개선에 있어서 두 중재 사이에 차이를 보 이지 않았다. ${ }^{8)}$ 따라서 정신의학적 교육이 인지행동치료만큼의 효과를 보이는 것으로 생각할 수 있다. 또한 다른 성인 $\mathrm{ADHD}$ 개방연구에서는 환자에 대한 정신의학적 교육을 실시한 결과, 환자의 질환에 대한 이해도가 향상되었고 아울러 사회생활에 서의 관계 개선 및 심리적인 만족감이 증가하였다고 보고하였 다." 따라서 향후에 더 많은 RCT 연구들을 통해 효과를 검증 해 보아야 할 것이나 성인 $\mathrm{ADHD}$ 의 경우에도 정신의학적 교육 프로그램이 도움이 될 수 있다는 가능성이 제기되고 있다. 성인 $\mathrm{ADHD}$ 의 경우는 정신의학적 교육의 일반적인 방법에 근거하 여 시행하면 되겠으나 $\mathrm{ADHD}$ 의 원인에 대한 설명을 통해 질 환에 대한 환자의 이해를 돕고, 핵심 증상 외에도 문제해결능 력이나 계획성 부분 등에서 나타날 수 있는 어려움에 대해 충 분히 설명해주는 것이 도움이 된다. 
정신의학적 교육은 $\mathrm{ADHD}$ 환자들의 증상, 치료 순응도, 주 관적인 만족도 등의 개선에 있어서 도움이 되는 효과가 있으 며, 따라서 정신의학적 교육은 $\mathrm{ADHD}$ 의 비약물치료 옵션에 서 충분히 고려할 만한 가치가 있는 것으로 보인다. 다만 이제 까지 시행된 연구들이 환자보다는 부모를 대상으로 이루어진 것들이 많고 성인 $\mathrm{ADHD}$ 에 대해서는 아직 연구가 초기 단계 에 머물러 있으므로 이에 대한 연구 결과들이 더 보완되어야 할 것이다. 또한 청소년과 교사를 대상으로 한 정신의학적 교 육 연구도 부족한 실정으로 이에 대한 충분한 연구 결과도 필 요할 것이다.

\section{행동적 부모훈련(Behavioral parent training, BPT)}

부모훈련은 부모관리훈련(parent management training) 혹은 행동적 부모훈련(BPT)으로 불리기도 한다. ${ }^{10)}$ 프로그램 목적은 $\mathrm{ADHD}$ 가 있는 학령전기 혹은 학령기 아동의 보호자에 게 긍정 강화 기법을 가르침으로써 부모양육행동을 변화시키 는 것이다. 이 프로그램은 사회학습이론을 바탕으로, 조작적 학습과 실용행동분석에 토대를 두고 있다. 부모의 양육방식 과 아동의 문제행동 간의 연관이 있으며 부모의 비일관적 양 육, 지도감독의 소홀, 방치 등이 문제행동을 증가시킬 수 있으 며 일관적이고 지지적인 부모의 개입, 안정적인 양육행태가 문 제행동을 줄일 수 있는 것으로 알려지며 이론적 배경이 되었 다. 프로그램의 목적은 ADHD가 있는 학령전기 혹은 학령기 아동의 보호자에게 긍정 강화 기법을 가르침으로써 부모양육 행동을 변화시키는 것이다. 부모훈련은 $\mathrm{ADHD}$ 증상뿐만 아
니라 아동의 외현화 증상과 적대적 반항장애, 품행 장애 등을 치료 목표로 시행되기도 한다. ${ }^{11}$ 부모훈련의 효과성은 다수의 연구에서 입증되었다. ${ }^{12)} 16$ 개의 연구(1003명의 학령전기 ADHD 아동)를 포함한 메타분석 결과, 부모훈련은 0.51 의 효과크기 (effect size)가 확인되었고, 독립적 관계를 분석했을 때 부정 적 양육방식과 통계적으로 유의미한 관련을 보였다. ${ }^{13)}$ 코크란 리뷰(Cochrane review)에서는 부모훈련이 아동의 품행 문제, 부모의 정신건강과 양육기술을 향상시키는 것으로 보고하였 다. ${ }^{14)}$ 또한 부모훈련 이후 부모의 양육 스트레스가 감소하고 주 관적 양육 효능감이 증가하는 것으로 확인되었다. ${ }^{15)} \mathrm{Kazdin}^{16)}$ 이 개발한 부모훈련은 주 1 회 간격의 12 회기 세션으로 구성되 어 있다. 각각의 회기에서는 아동의 문제행동을 확인, 목록화 하고 그에 대한 대응 행동을 결정하고 이를 실천하는 것으로 이루어져 있다. 아동의 행동에 대해 사용할 수 있는 개입 기 법들은 다음과 같다(Table 1). 최근에는 부모훈련에서 흐름도 를 사용하는 것이 도움이 될 수 있다는 제안도 있다(Fig. 1). ${ }^{17}$ $\mathrm{ADHD}$ 아동의 보호자 역시 $\mathrm{ADHD}$ 의 특성을 보일 가능성 이 높다. 보호자가 $\mathrm{ADHD}$ 를 진단받거나 역치하(subthreshold) 의 $\mathrm{ADHD}$ 특성을 보일 때 부모훈련의 효과가 감소한다는 보 고가 있다. ${ }^{18)} \mathrm{ADHD}$ 를 진단받은 엄마를 대상으로 다양한 방법 (multimode)의 치료를 진행했을 때 더 효과가 컸다는 보고도 있다. ${ }^{19)}$ Chronis-Tuscano 등 ${ }^{20)}$ 은 ADHD가 있는 보호자 대상 부모훈련과 부모 약물치료를 통합한 맞춤형 치료의 효과성을 검증하는 연구를 진행 중이다. 부모의 반사회성 특성이 부모 훈련의 효과성을 감소시킬 가능성도 제기되었다. ${ }^{21)}$ 부모훈련

Table 1. Behavioral intervention techniques used in parent training

\begin{tabular}{|c|c|}
\hline Interventions & Examples of intervention methods \\
\hline \multicolumn{2}{|l|}{ Positive interventions } \\
\hline Point program & $\begin{array}{l}\text { Used for any positive behavior you want to increase (focus on up to three behaviors at a time), } \\
\text { such as minding, getting along with a sibling, talking nicely, doing any chore, potty training. }\end{array}$ \\
\hline Praise program & $\begin{array}{l}\text { Used for increasing any behavior (which happens more than once a day), such as talking politely, } \\
\text { playing nicely, helping out, and listening to instructions, solely through the use of your attention and } \\
\text { praise. }\end{array}$ \\
\hline Shaping & $\begin{array}{l}\text { Used with any behavior you can break down into smaller parts to reinforce the smaller parts } \\
\text { individually, such as picking up toys, going to bed on time, feeding a pet, putting dirty clothes in the } \\
\text { laundry, and getting dressed on time. }\end{array}$ \\
\hline Attending & $\begin{array}{l}\text { Used in conjunction with ignoring (giving attention to the positive opposite of the behavior you are } \\
\text { ignoring), such as any positive opposite of the ignored behavior. }\end{array}$ \\
\hline \multicolumn{2}{|l|}{ Negative interventions } \\
\hline Time out & Used with verbal or physical aggression, such as hitting, pushing, punching, talking back, or swearing. \\
\hline Ignoring & $\begin{array}{l}\text { Used with mildly annoying behaviors that do not need to be stopped right away, such as whining, } \\
\text { interrupting, complaining, having an attitude, or pleading. }\end{array}$ \\
\hline Reprimands & $\begin{array}{l}\text { Used with behaviors that parents decide need to be stopped right away, such as jumping or running } \\
\text { in the house, yelling, and being loud. }\end{array}$ \\
\hline Low-rate program & $\begin{array}{l}\text { Used with behaviors that occur no more than two or three times a week and are serious in nature, } \\
\text { such as stealing, lying, property destruction, wandering away, and playing with matches. }\end{array}$ \\
\hline
\end{tabular}




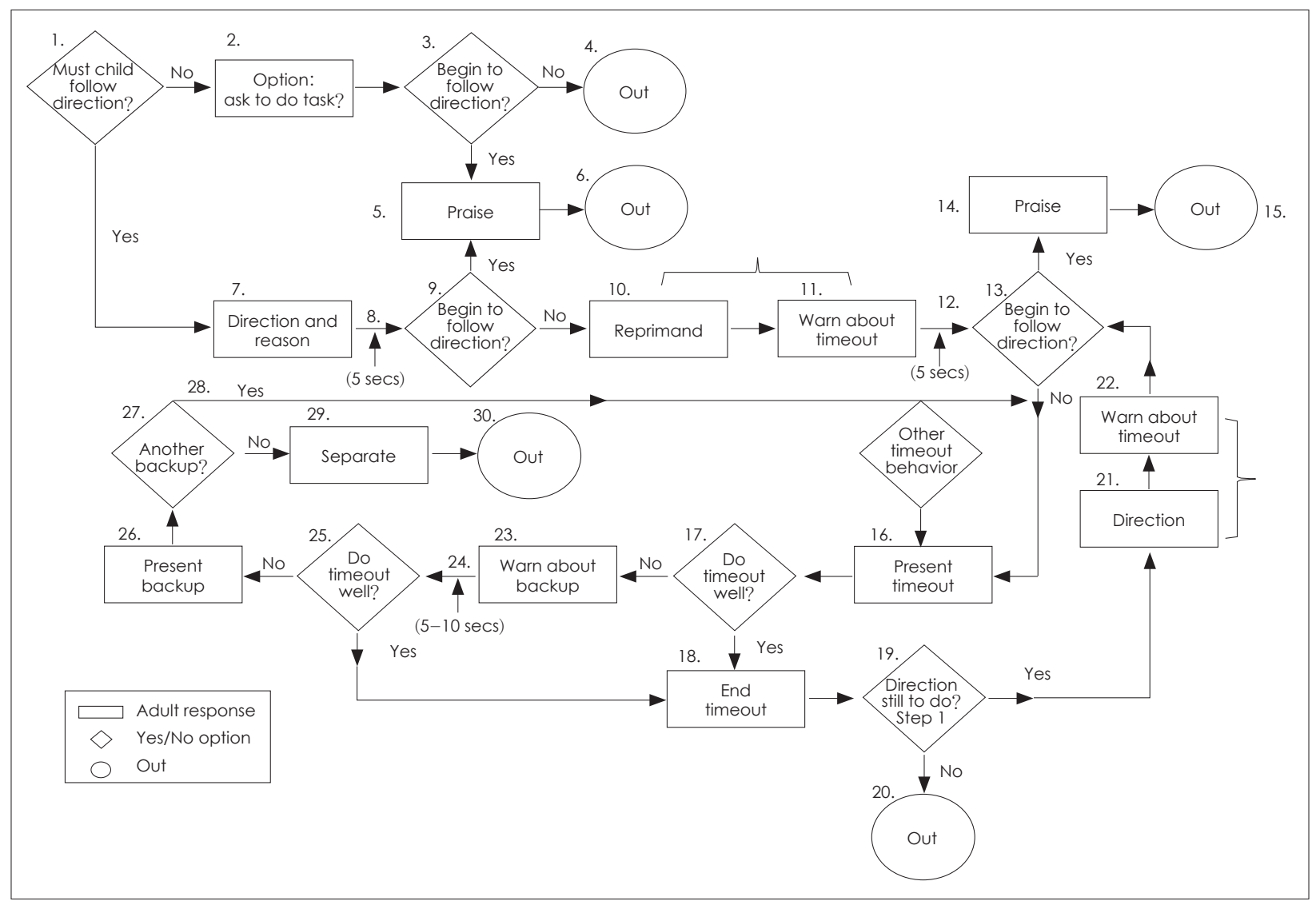

Fig. 1. An example of a Behavior Management Flow Chart.

에 사회기술훈련이나 작업기억력훈련 등 다른 행동적 치료기 법을 병행하는 게 도움이 된다는 연구가 발표되고 있다. ${ }^{22)}$ 이 란에서는 부모훈련과 작업기억력훈련을 함께 시행했을 때, 각 각의 치료를 단독으로 시행했을 때에 비해 $\mathrm{ADHD}$ 증상의 감 소가 더 컸던 것으로 보고하였다. ${ }^{23)}$ 대만에서 학령기 아동을 대상으로 8 주간 부모훈련과 사회기술훈련을 함께 진행한 결 과 치료 후 반항적 행동 및 자기 조절력 등에서 유의미한 호 전이 있는 것으로 보고하였다. ${ }^{24)}$

\section{특수교육적 개입}

\section{행동 개입}

행동 개입은 주의력과 행동을 향상시키는 전략으로, 사회적 으로 바람직하지 않은 행동을 사회적으로 적절한 행동으로 바꾸는 것을 목표로 한다.

1) 교사-학생 관계 형성: 강한 교사-학생 관계는 학생이 학 교 활동에 참여하고, 규칙을 따르고, 또래와 효율적으로 관계 하기 쉽게 하는 상황을 조성한다. 25$)$

2) 규칙 게시하기: 공통된 학급 규칙을 게시하는 것은 행동 에 대한 기대를 명확히 한다. ${ }^{26}$
3) 효과적인 지시/명령: 아동이 무엇을 해야 할지에 대한 간 결한 선언문이다. 명령은 한 번 내려지고, 보상 또는 주의가 따 라야 하며, 비순응에 대해서 적절한 결과가 있어야 한다. ${ }^{27)}$

4) Contingent attention: 적응적인 행동에 대한 반응으로 칭 찬과 함께 교사의 관심을 제공하는 것은 행동을 바꾸는 효과 적인 전략이다. ${ }^{28)}$

5) 구체적인 강화: 적응적인 행동에 대한 구체적인 강화물 을 제공하는 것은 강화체계를 더 튼튼하게 한다. 이후 보상과 맞바꿀 수 있는 점수를 주는 것은 실행가능성을 증가시킬 수 있다. ${ }^{29)}$

6) 반응 비용: 비적응적인 행동에 반응해서 점수를 깎는 것은 점수 강화체계나 토큰에 유용한 부가적 방법이 될 수 있다.29)

7) 전략적 언어 교정: 행동에 뒤따르는 간단하고 직접적인 언어적 교정은 긍정적 강화체계에 효과적인 부가적 방법이 될 수 있다. ${ }^{30)}$

8) 가족-학교 파트너십 형성: 강한 파트너십 상황 안에서, 교 사와 부모는 학생의 강점을 강화하고 상호 간의 문제해결에 개입할 수 있다. ${ }^{31}$

9) 일일 행동 카드(daily report card): 한 개 이상의 정해진 목표에 대해서 교사가 아이에 대해 평정하는 것이다. 그리고 부 
모는 받은 점수를 기반으로 보상을 제공할 수 있다. ${ }^{32)}$

\section{학습 개입}

학습 개입 전략은 증상과 관련된 기능적 손상에 초점을 맞 춘다. 읽기와 산수 실력을 높이는 것뿐만 아니라 학습과제에 대한 수행을 향상시키는 것을 목표로 한다. 학습 기술 습득과 $\mathrm{ADHD}$ 로 인한 학습과제수행의 어려움을 다루기 위한 개입 에는 명확한 지시, 컴퓨터 지원을 이용한 지시, 또래 교습(peer tutoring)이 포함될 수 있다.

학습개입은 무엇을 배워야 할지에 관해서 학생들에게 분명 한 정보를 제공하고, 구체적이고 다양한 실례를 이용하여 소 단계로 기술을 가르치는 것과 학생들의 이해도를 꾸준히 파 악하여 적극적인 참여를 이끌어 내는 것을 목표로 하고 있다.

1) Instructional match: 학생의 현재 능력에 맞춘 수준의 교 육을 시행하는 수행 관찰은 학습 수행을 향상시킬 수 있다. ${ }^{33)}$

2) 적극적인 참여: 신기하고 흥미롭고 적극적인 참여를 허용 하는 과제는 학습 참여도를 높인다. ${ }^{34)}$

3) 목표 설정: 학생이 작업완료, 정확성, 시간, 자기-평가 수 행에 대한 목표를 설정하는 데 적극적으로 참여하도록 하는 것은 시간관리, 작업 완수와 정확도를 향상시키는 데 효과적 인 방법이다. ${ }^{35)}$

4) 또래 교습: 개별화된 지시와 빈번한 피드백 기회를 제공 한다. ${ }^{30)}$

5) 부모 교습: 지시에 대한 기회를 증가시키고, 학생이 학교 에서 제공된 수업을 달성하는 것을 돕는다. ${ }^{37)}$

6) 컴퓨터 지원형 전략(computer assisted strategy): 호감을 유발하는 학습자료, 학습목표 및 노력과 수행에 대해서 즉각 적인 강화를 주는 것을 결합할 수 있다. ${ }^{38)}$

\section{자기 조절력 개입}

자기 조절력 개입은 기능적 장애를 경험하는 환경에서 자기 조절력을 높이기 위해 노력하는 능력을 향상시키도록 하는 것에 목표를 둔다. 자기-감지 개입은 학생이 끊임없이 목표 행 동을 확인하도록 하고, 이러한 행동의 일부를 기록하도록 가 르치는 것으로부터 시작한다.

1) 목표 행동을 선택

2) 기록 방법을 선택

3) 학생의 자기-감지의 정확성을 평가할지를 결정

4) 강화와 관련된 결정을 내림

\section{사회적 효능감 향상 전략}

$\mathrm{ADHD}$ 아동은 친구를 사귀고 유지하는 것에 대한 어려움과 또래로부터 배제당하는 것과 같은 또래관계 문제를 겪는다. ${ }^{39)}$
또래관계가 좋지 않은 것은 부정적인 영향을 미치고, 아이들에 게 심각하게 부정적인 결과(물질 남용, 학교 중퇴)를 초래할 수 있다. 따라서 또래집단의 구성원을 개입 과정에 포함시키는 것이 중요하다. ${ }^{40)}$

1) 사회기술훈련: 친사회적 행동을 가르치고 강화하는 것이 고, 사회적 정보를 처리하는 데 어려움이 있는 학생들에게 도 움이 될 수 있다. ${ }^{41)}$

2) 지속적인 친구관계 증진: 집중적인 행동치료프로그램을 하는 과정에서 아이들의 우정이 잘 유지되도록 도움으로써 아 이들의 반사회적 행동을 줄일 수 있다. ${ }^{42)}$

3) 점심시간 개입: 집단의 목표를 달성하면 그 집단의 모든 학생에게 긍정적 강화를 제공함으로써(집단 강화, group contingency), 점심시간 행동을 개선시킬 수 있다. ${ }^{43)}$

4) 운동장 개입: 조직화된 게임을 도입하고, 이에 대한 적절한 감독을 하며 친사회적 행동을 강화함으로써 적절한 사회적 행 동을 촉진시킬 수 있다. ${ }^{44}$

\section{인지행동치료(Cognitive-behavioral therapy, CBT)}

인지행동치료는 새로운 사고 체계를 이용하여 기저의 부정 적 사고와 신념을 바꾼다는 전제를 바탕으로 이뤄지는 정신 치료의 한 종류이다. 성인에서 정신건강문제를 치료하고 대처 기술 개발을 하는 데 유용한 것으로 알려져 있다. 학령기 아동 의 기분장애와 불안장애에서는 인지행동치료가 활발히 이용 되고 있으며, 성인과 청소년 $\mathrm{ADHD}$ 에서도 인지행동치료가 유 용하다는 최근 연구들이 있다. ${ }^{30,45,46} \mathrm{ADHD}$ 아동의 경우 증상 이 심하지 않고 약물치료에 반응이 없는 경우 시행하게 된다. 인 지행동치료의 구체적 방법으로 모델링, 역할극 등을 통해 문 제해결능력을 키우기도 하고 자기 지시훈련을 통해 자신의 행 동을 조절하는 기술을 익히게 된다. 학령기 아동의 경우, 1991 년 발표된 메타분석에서 문제행동과 사회적 부적응이 있는 $\mathrm{ADHD}$ 아동에서 cognitive-behavioral therapy(CBT)가 도움 이 될 수 있다고 발표되었고, ${ }^{47)}$ 최근 RCT 연구에서는 $\mathrm{CBT}$ 가 $\mathrm{ADHD}$ 아동의 핵심 증상에 유망한 치료라고 보고된 바 있 다. ${ }^{48)}$ 가장 최근에 발표된 아동 $\mathrm{CBT}$ 에 관한 메타분석에서 $\mathrm{ADHD}$ 증상의 호전에 대해 중등도 효과크기(moderate effect size)가 있다고 보고하였다. ${ }^{49)}$ 아동에 대한 CBT 유용성에 관해서는 논란이 있어 왔지만,50) 최근 보고된 결과들은 아동 에 대한 CBT를 지지하는 경향으로 보인다. 하지만, 앞으로 장 기간의 효능 및 안전성에 대한 연구가 뒷받침되어야 할 것으 로 보인다. ADHD 아동의 경우 증상이 심하지 않고 약물치료 에 반응이 없는 경우 인지행동치료를 시행하게 된다. 인지행동 치료의 구체적 방법으로 모델링, 역할극 등을 통해 문제해결 능력을 키우기도 하고 자기 지시훈련을 통해 자신의 행동을 
조절하는 기술을 익히게 된다. 하지만 앞으로 장기간의 효능 및 안전성에 대한 연구가 뒷받침되어야 할 것으로 보인다.

\section{사회성훈련프로그램}

많은 $\mathrm{ADHD}$ 아동이 사회적 상호작용에 문제가 있어서, 부 모를 비롯한 가족, 친구, 선생님과의 관계 형성에서 부정적 경 험을 하게 된다. 약물치료는 $\mathrm{ADHD}$ 아동의 주요 행동 증상 의 조절에 도움이 되지만 사회성의 부족을 개선시키기에는 부족하기 때문에 사회기술훈련이 부가적으로 필요하게 된 다.1) '사회기술(social skills)'이라는 용어는 개인이 효과적으로 타인과 상호작용하기 위하여 사회적으로 수용되는 모든 행동 을 의미한다. 사회기술에는 사회적인 상호작용을 방해하는 문 제행동(problematic behavior)과 사회적 상호작용을 보다 효과 적이게 하는 친사회적 기술(pro-social skill)이 포함된다. 친사 회적 기술의 예로는 친구들과 협력하기, 친구 사귀기, 갈등 상 황에서 분노 조절하기 등이 있다. ${ }^{52)}$ 사회기술은 성공적인 삶을 살아가는 데 필수적이며 학업 성취, 친구 및 가족 관계, 직업을 유지하는 데 중요한 영향을 준다. ${ }^{53)}$ 사회기술이 부족한 아동들 은 학교를 입학하기 전부터 정서적, 행동적 문제가 나타나며, 적절한 개입이 이루어지지 않을 경우 성인기까지 이러한 문제 가 지속될 수 있다. ${ }^{54)}$ 사회기술이 부족한 아동은 학교 적응에 어려움을 보여 자퇴, 유급, 탈선 행동이 증가하고, 사회기술의 부족은 청소년기의 우울, 불안, 반사회적 행동의 증가와 관련 된다.55,50) 적절한 사회기술의 형성은 사회성 발달에 필수적인 역할을 한다. 아동이 매일 경험하게 되는 사회적 맥락에 적응하 기 위해서 아동은 사회적인 상황을 정확히 인식하여 상황에 적합한 사회기술을 사용할 수 있어야 한다. 효과가 입증된 프 로그램을 통하여 교육으로 습득할 수 있다. 1970년대부터 사회 기술훈련이 개발되어 시행되고 있다. ${ }^{57}$ 사회기술훈련은 인지행 동치료의 한 종류로서 치료자에 의해 개별적으로 아동에게 교육할 수도 있으나, 그룹 형태를 구성한 상태에서 기술들을 전달함으로써 아동이 직접적으로 그들의 사회적 기술을 연습 해 보는 기회를 제공할 수도 있다. 아동들은 사회적 상호 관계 속에서 그들의 언어적, 비언어적 행동들을 어떻게 적용할 것 인가를 배우게 되고, 세상에 대한 인지적인 평가 방식을 바꾸 려는 노력을 포함한다. 훈련은 차례를 기다리거나, 대화 중 주 제를 바꾸는 시기나 다른 이들의 감정적 표현을 인지하는 것 과 같은 사회적 상호 관계에서 나타나는 미묘한 신호를 읽어 내 는 방법을 가르치는 데 중점을 둔다. ${ }^{58)}$ 또한 사회적으로 평범 한 것들, 사회적 규범, 다른 이들의 기대 등을 가르친다.59) 정서 기술(emotional skills)은 아동이 자신의 감정을 다루고, 표현하 고, 조절하는 능력에 관한 것으로 자기 정서조절은 회복탄력 성의 중요한 요소이다. 실망, 상실을 비롯하여 당황스러운 사건
을 효율적으로 다룰 수 있는 아동은, 그렇지 않은 아동에 비해 심리적 어려움에서 회복할 가능성이 높다. 사회기술훈련은 다 음의 프로그램들로 구성되며 이를 기반으로 한 다양한 변형들 이 적용되고 있다. ${ }^{60)}$

1) 사회기술 행동 훈련(behavioral social skills training): 지침, 토의, 모델링, 역할극, 리허설, 강화, 피드백 등

2) 사회인지 훈련(social perception skills training): 사회적 맥락에서 사회적인 신호를 정확하게 해석하기 등

3) 자기조절/자기 지시 기술(self-regulation/self-instructional techniques): 자기관찰(self-monitoring), 자기대화(selftalk), 자기강화(self-reinforcement) 등

4) 사회적 문제해결능력(social problem solving): 문제 확 인, 대안 찾기, 결과 예측, 적절한 반응의 선택과 계획 등

5) 경쟁, 억제, 부적절한 반응의 감소(reduction of competing/ inhibiting/inappropriate social response): 부모교육, 우발상황 관리(contingency management), 이완 훈련, 인지 재구조화 등

치료회기 내의 수행을 통해 임상가는 아동의 행동을 바로 잡아주며 아동의 성공적인 기술을 사용하는 것을 강화하도 록 한다. 치료의 효과는 사회기술 자체를 관찰하거나, 교우관 계, 정서조절 능력, 평상시 행동과 같은 심리적 기능의 전반적 인 평가를 통해 판정한다. 사회기술훈련을 적용하는 데 있어 연령이 중요한 조절 변수로 작용하기 때문에 청소년에 비해 아 동에서 더 효과가 있다고 알려져 있다. ${ }^{61)}$ 사회기술훈련이 효과 적으로 시행되고 치료적인 변화를 일으키기 위해서는 양육방 식, $\mathrm{ADHD}$ 의 아형, 공존 질환, 연령 등의 요인들이 영향을 미 친다. ${ }^{62}$ 최근 10 년 동안 여러 대안적인 사회기술훈련 기법들이 개발되고 있다. 이러한 대안적 사회기술훈련 기법들은 실제 생 활에서 아동들이 친구들과 상호작용하는 상황에 즉각적인 피 드백을 줄 수 있도록 아동의 부모나 교사들을 훈련시키는 데 조금 더 초점을 맞추고 있다. 전통적인 사회기술훈련에 부모 훈련을 추가하는 것이나, ${ }^{63)}$ 놀이에 기반한 사회기술훈련이 사 회적 의사소통능력의 향상에 도움을 주며, ${ }^{64)}$ 음악 치료에 기 반한 사회기술훈련이 ADHD 아동의 사회기술을 향상시킬 수 있다. ${ }^{65)}$

\section{부모-아동 상호작용치료}

부모-아동 상호작용치료(Parent-Child Interaction Therapy, $\mathrm{PCIT}^{66)}$ 는 부모가 관련 기술들을 배우고 직접 연습할 수 있 도록 구조화되어 있다. PCIT는 애착이론과 사회학습이론을 바탕으로 개발되었으며, 두 단계로 이루어져 있다. 첫 번째 단 계는, 관계 개선 단계로 아동주도 상호작용(child-directed interaction)이 이루어지고, 두 번째 단계는 행동관리 훈육 단 계로 부모주도 상호작용(parent-directed interaction)이 이루 
Table 2. PRIDE skills in parent child interaction therapy

\begin{tabular}{cl}
\hline Initial & \multicolumn{1}{c}{ Explanation } \\
\hline P & Praising the child \\
R & Reflecting the child's behavior \\
I & Imitating the child \\
$D$ & Describing their child's behavior \\
E & Using enthusiasm \\
\hline
\end{tabular}

어진다.

첫 번째 단계는 정통적인 놀이치료와 유사하다. 정통적으로 놀이치료는 아동이 놀이를 통해 자신의 감정과 경험을 표현 하게 함으로써 아동이 중심이 되어 자연스러운 치유를 돕는 과정이다. ${ }^{67)}$ PCIT에서는 이 단계에서 부모가 PRIDE 기술을 사용하도록 교육받는다(Table 2).

두 번째 단계에서는 부모가 단호하고 일관되게, 예측 가능 한 훈육을 할 수 있도록 교육한다. 이 단계는 부모훈련과 유사 하나, 부모훈련에서는 생물학적 질병으로서의 $\mathrm{ADHD}$ 에 초점 을 맞춰, 질환에 대한 정신의학적 교육을 포함한 10 회기의 프 로그램이 이루어지는 반면, PCIT에서는 기술 습득에 초점을 맞춰 회기 횟수를 미리 정하지 않고, 기술을 충분히 익혔다고 생각될 때까지 교육과 연습이 진행된다는 점이 특징이다.

PCIT는 여러 연구를 통해 어린 아동에서 비적응적 문제행 동을 감소시킨다는 점이 확인되었다. 대조군 비교 연구를 통 해 PCIT 치료를 받은 아동에서 문제행동이 감소할 뿐만 아니 라, 학교 환경에서도, 그리고 치료받지 않은 형제에서도 긍정 적인 영향이 있다는 결과 또한 보고된 바 있다. ${ }^{68)}$ 또한 PCIT 사전사후 검사를 통해 $\mathrm{ADHD}$ 증상이 감소되는 것은 물론, 장기적으로 그 효과가 유지된다는 보고도 있다. ${ }^{66)}$

\section{놀이치료}

$\mathrm{ADHD}$ 에 대한 놀이치료의 의학적 근거는 아직 부족하기 때 문에, 놀이치료는 기존의 $\mathrm{ADHD}$ 임상 치료 진료지침에서 추 천되고 있지 않다.169) 하지만, 정서적 어려움과 사회성 문제가 동반된 $\mathrm{ADHD}$ 아동에서는 놀이를 이용한 개입이 도움될 수 있다. ${ }^{70)}$ 놀이치료는 아동의 발달에 적합하며, 아동의 내재적이 고, 외현적인 문제행동을 개선하는 데 효과적인 것으로 보고 되어 왔다. ${ }^{71)}$ 이를 바탕으로 놀이에서의 상호작용, 규칙, 경쟁, 목표 지향적 특성들이 $\mathrm{ADHD}$ 아동에 긍정적 영향을 미칠 것 으로 기대하여, 다양한 놀이치료들이 행해지고 있다. 게임놀이 치료에서 주로 사용되는 게임의 종류에는 주의력과 관련해서 는 다트게임, 낚시하기, 도미노, 블록 쌓기 등이 있고, 충동조 절 및 자기통제력과 관련해서는 STOP 보드게임, 얼음 땡 게임 등이 있다. ${ }^{72)}$ 인지행동놀이치료는 인지행동치료에 놀이치료적 요소들이 결합된 형태로서, 모델링, 역할놀이 등의 행동적 개
입과 부적응적 생각의 증거를 찾고 대안을 탐색하는 인지적 방법들이 사용된다. 아동중심놀이치료는 증상보다 아동 자체 에 초점을 두고 있다. 아동중심놀이치료는 아동 스스로 성장 하고자 하는 힘과 치료적 방향을 믿고 진행하는 비지시적인 놀이치료로서 $\mathrm{ADHD}$ 아동에서도 효과적일 수 있다. ${ }^{73)}$ 게임놀 이치료와 인지행동놀이치료가 $\mathrm{ADHD}$ 아동의 행동변화에 적 용될 수 있다는 점을 일부 연구들에서 보여주고 있으나, 구체 적인 치료적 과정과 기전에 대한 의학적 연구는 아직까지 미미 한 수준이다. 그 외에도 모래놀이치료, 통합놀이치료, 가족놀 이치료, 심리운동놀이치료 등이 $\mathrm{ADHD}$ 아동의 사회성과 정서 적 어려움을 도와주기 위해서 시도되고 있으나, 향후 이들에 대한 의학적 연구와 고찰이 필요할 것이다.

\section{성인기 ADHD 인지행동치료}

$\mathrm{ADHD}$ 인지행동치료는 성인에서 기분장애나 불안장애 등 에 탁월한 효과가 있으며 성인 $\mathrm{ADHD}$ 에도 효과가 있다는 보 고들이 지속되고 있다. 청소년에게도 도움이 될 수 있으나, ${ }^{74)}$ 인 지행동치료는 동기와 변화에 대한 신실한 약속이 필요한데 청소년에서는 부족할 수도 있는 부분이므로 성인에서보다는 그 효과가 현저하지 않을 수 있다. ${ }^{75-77)}$ 성인의 경우 인지행동치 료의 적응증은 다음과 같다.

1) 약물치료를 받지 않는 사례에서 그 장단점과 한계에 대 한 설명을 듣고 의논하여 인지행동치료를 받기로 결정한 경 우, 2) 약물치료에 효과가 충분하지 않거나 투약에 견디기 어려 운 경우로 판단된 경우거나, 3) $\mathrm{ADHD}$ 라는 진단을 받아들이기 어려워하고 약물치료를 지속적으로 받는 것이 어려운 경우, 4) 증상이 관해되었지만 경도나 중등도의 기능상 문제를 인지행 동적 접근의 목표로 잡아도 충분한 경우에 고려할 수 있다. ${ }^{2}$ 인지행동치료의 예를 들면, 문제가 되는 인지와 행동에 초점 을 맞추고, 이를 평가 시에 확인하고 생각을 기록하거나 행동 적인 실험을 통해 근거 기반의 체계화된 공식을 사용하여 진 행한다. 시간관리 등과 같은 기술에 기반을 둔 다양한 접근을 하게 되는데 치료 기간과 시간, 내용 등의 $\mathrm{CBT}$ 매뉴얼은 연 구자에 따라 관점의 차이가 있어 ${ }^{78)}$ 환자에게 보다 적합한 근거 기반의 접근을 하는 것이 필요하다. 각기 다른 성인 $\mathrm{ADHD}$ 의 인지행동적 치료의 접근 방법들을 분류해서 정리하였다 (Table 3).

\section{기타: 코칭(Coaching), 보완대체의학, 뉴로피드백, Cogmed}

\section{코칭(Coaching)}

코칭은 환자의 일상생활을 파악한 후 환자들이 자신의 목 표를 달성할 수 있도록 도와주는 것이다. 성인 $\mathrm{ADHD}$ 환자들 
은 발병 시기, 임상 양상, 처한 상황 등이 모두 다르기 때문에 개인에게 미치는 병의 영향은 다양하다. 따라서 코칭에서는 환 자의 인생에서 질환이 어떠한 영향을 미쳐 왔고 현재 어떠한
영향력을 미치고 있는지 자세한 평가가 필요하다. 이러한 평 가를 통해서 개인에게 맞춘 치료 전략을 구상할 수 있다. 이 를 도와주는 치료자를 코치라 부르게 되며 코치는 환자의 기

Table 3. Components of CBT for adult ADHD

\begin{tabular}{|c|c|}
\hline Module & Dpecific skills \\
\hline \multirow[t]{2}{*}{ Psychoeducation } & Education about ADHD symptoms in adulthood \\
\hline & Involve family members \\
\hline \multirow[t]{2}{*}{ Organization and planning } & Prepare tools for planning and organize (appointment handbook, assignment list note) \\
\hline & handling multiple kassignments, prioritizing \\
\hline \multirow{2}{*}{$\begin{array}{l}\text { Gauging attention span and } \\
\text { distractibility delay }\end{array}$} & Measuring attention time (using stop watch) \\
\hline & Attention distributed delayed training method \\
\hline \multirow[t]{3}{*}{ Modifying the environment } & $\begin{array}{l}\text { Work environment control: analyze and control distracting factors (e-mail, messages, } \\
\text { windows, etc.) }\end{array}$ \\
\hline & Locating important things: keys, wallets, notes \\
\hline & Use a tool to remind of your attention: sticker \\
\hline \multirow[t]{3}{*}{ Managing overwhelming tasks } & $\begin{array}{l}\text { Problem-solving process: identify the problem accurately, draw out possible alternatives, } \\
\text { list the alternatives, list the disadvantages, evaluate the alternatives, and implement the } \\
\text { best alternative }\end{array}$ \\
\hline & The big task is to break it down into several manageable steps \\
\hline & Systematically organize your filing \\
\hline \multirow[t]{3}{*}{ Cognitive restructuring } & Understanding automatic negative thinking \\
\hline & Understanding and applying the errors of thinking (dichotomous thinking, \\
\hline & over-generalization, etc.) \\
\hline \multirow[t]{4}{*}{ Reducing procrastination } & Adaptive thinking for deferring behaviors: list the situations that lead to deferred \\
\hline & behaviors, list automatic thinking about tasks or lists, and identify feelings associated \\
\hline & with an accident. Identify accidental errors \\
\hline & Adaptive thinking to deal with postponing behavior \\
\hline Anger and frustration management & Relationship problems, anxiety, anger control, depression. sleep, substance abuse \\
\hline
\end{tabular}
ADHD: attention-deficit hyperactivity disorder, CBT: cognitive-behavioral therapy

Table 4. Complementary and alternative medicine in ADHD

\begin{tabular}{|c|c|}
\hline CAM & Key points \\
\hline Feingold diet & No evidence. \\
\hline Sugar and aspartame acid & No evidence. Restricted diets are not recommended by AACAP. \\
\hline $\begin{array}{l}\text { Artificial colors and } \\
\text { preservatives }\end{array}$ & $\begin{array}{l}\text { Causal relationship unclear. Some problem behaviors may be noticeable among children who } \\
\text { are sensitive to food additives. }\end{array}$ \\
\hline Vitamin & No evidence. Mega vitamin therapy has hepatotoxicity, neurological toxicity risk. \\
\hline Zinc & $\begin{array}{l}\text { If the zinc intake in children with ADHD is insufficient, it is recommended to encourage adequate } \\
\text { intake or to use it after preliminary blood test. }\end{array}$ \\
\hline Iron & Consistent results do not appear. The main treatment is lack of evidence. \\
\hline Magnesium & $\begin{array}{l}\text { Some studies have improved symptoms. No randomized, double-blind, placebo-controlled } \\
\text { studies were performed. After checking the concentration, take it if necessary. }\end{array}$ \\
\hline Omega-3 & $\begin{array}{l}\text { Studies have been conducted since 2005. In two of the three review article, Omega-3 was } \\
\text { found to be mild but effective. Not recommended for monotherapy. }\end{array}$ \\
\hline Amino acid supplement & $\begin{array}{l}\text { Short-term tryptophan, tyrosine, and phenylalanine supplements were effective, but the effect } \\
\text { for 2-3 months was not proven. Risk of long-term use. }\end{array}$ \\
\hline Antioxidant, herbal medicine & $\begin{array}{l}\text { Pycnogenol: two control studies were performed, but no effective. } \\
\text { Kava kava: not recommended. } \\
\text { Chinese medicine, acupuncture: open research only. }\end{array}$ \\
\hline
\end{tabular}

AACAP: American Acedemy of Child and Adolescent Psychiatry, ADHD: attention-deficit hyperactivity disorder, CAM: complementary and alternative medicine 
상시간, 약 먹는 시간, 회의 시간, 마감시간 등을 체크해서 환 자에게 알려주고 문제가 생기지 않도록 도와준다. 이외에 일 상에서 벌어지는 일들을 계획하고 처리하고 체계화하는 것 등을 도와주게 된다. 코칭 시스템은 미국에서 활성화되어 있 고, 코치는 성인기 $\mathrm{ADHD}$ 문제들을 도와주기 위해서 전문적 으로 훈련된 사람을 말하게 된다. 국내의 경우 전문 코칭 시 스템이 없기 때문에 가족이나 친구, 치료자들이 이러한 역할 을 어느 정도 대신해 줄 수 있다. 코치와 환자는 조언을 벗어 나 문제해결을 위해 같이 협력하는 목표 지향적 관계이다. $\mathrm{ADHD}$ 성인의 경우 삶의 다양한 영역에서 선택을 하게 되는 데, 잘못된 선택은 오랜 기간 적응의 문제를 일으키게 되기 때 문에 코칭은 이러한 영역의 문제를 도와주게 된다.

보완대체의학(Complementary and alternative medicine, CAM)

전통의학을 대신하거나 기존 전통의학을 보완하고 통합하 자는 의미로 사용되고 있는 의학의 한 분야이다. 연구들에 따 르면 $\mathrm{ADHD}$ 아동의 경우 보호자가 약물치료에 대한 부담감 때문에 보완대체의학을 흔히 사용하고 있는 것으로 나타났으 며 한국의 경우도 마찬가지이다. 그동안 $\mathrm{ADHD}$ 에 영향을 미 치는 것으로 알려진 페인골드 식이, ${ }^{79)}$ 설탕 및 아스팜산, ${ }^{80)}$ 아 연, ${ }^{81)}$ 철, ${ }^{82)}$ 오메가 $3^{83,84}$ 등의 근거에 기반한 결과들에 대해서 정리하였다(Table 4).

\section{뉴로피드백}

뇌파를 이용한 바이오 피드백 훈련인 뉴로피드백은, 정신 상 태(mental state)를 반영하는 뇌파에 대한 실시간 피드백을 통하여 조작적 조건화 이론에 따라 뇌파를 변화시킴으로써, 보다 높은 수준의 자기조절능력(self-regulation)을 학습하는 훈련이다. 1976년 Lubar와 Shouse ${ }^{85}$ 에 의해 "sensorimotor Rhythm(SMR) 증가 훈련"을 통하여 ADHD 아동의 과잉행동 이 호전됨을 보고한 연구 논문이 최초로 발표된 이래 $\mathrm{ADHD}$ 아동을 대상으로 한 뉴로피드백 훈련의 치료 효과에 대한 연 구가 본격화되기 시작하였고, 2009년 Arns 등 ${ }^{80}$ 은 각각의 독립 적인 메타분석 연구를 통하여 뉴로피드백을 $\mathrm{ADHD}$ 치료에 있 어서 최고 수준의 효능을 가진 치료법(level 5 efficacy)이며, 부 주의 및 충동성의 치료 효능에 있어서 약물치료와 동등한 효 과크기(effect size)를 가진다고 주장하였다. ${ }^{87-92)}$ 그러나 최근의 메타분석 연구 결과들은 뉴로피드백의 효과를 입증하지 못하 였다.93,94) 향후 Randomized Controlled Study를 통하여 보다 많은 근거들이 뒷받침되어야 할 것이다. 또한 뉴로피드백 훈련 의 정확한 작용 기전에 대한 추후 연구들이 필요하다. 뉴로피 드백은, 의료 전문가들이 그 평가 기법과 훈련 기법 등 사용법 에 대한 교육 과정을 이수하여 적절한 훈련이 이루어진 상태에
서 사용하게 된다면 $\mathrm{ADHD}$ 치료에 있어서 잠재적인 치료 기법 으로 간주될 수 있을 것이다.

\section{Cogmed}

Cogmed Working Memory Training 프로그램은, 2005년 Klingberg 등 ${ }^{95)}$ 이 무작위대조군 이중 맹검 연구(randomized controlled double blind trial)를 통하여 ADHD 아동의 작업 기억력을 비롯한 신경인지 기능 향상 및 $\mathrm{ADHD}$ 증상 개선 효 과(부모 보고 평정척도의 향상)를 발표한 뒤로, 지난 10 년간 여러 연구들을 통해 그 치료적 효능이 검증되어 왔다.96) 현재 까지 내려진 결론은, 프로그램을 통하여 훈련된 언어적 비언 어적 작업기억력의 단순 저장(storage) 능력은 향상되지만, 복 합 작업기억력(complex working memory), 즉 저장과 더불어 처리/집행(storage plus processing/manipulation) 능력 향상에 는 영향이 없고, 훈련 효과의 일반화(generalization) 및 $\mathrm{ADHD}$ 증상 호전(부모 및 교사 평정척도)에 대한 근거 부족으로 아 직까지는 $\mathrm{ADHD}$ 에 대한 비약물적 치료법으로 인정받기에는 그 근거가 불충분하다. ${ }^{97)}$ 그러므로 작업기억력의 결핍이 현저 한 특정 $\mathrm{ADHD}$ 군이나, 인지적 유동성이 높은 학령전기 아동 을 대상으로 한 치료적 유용성에 대한 연구가 더 활발히 이루 어져야 할 것이다. ${ }^{98)}$

\section{권고사항}

권고사항 1. 환자와 가족들에게 정신의학적 교육을 시행해 야 한다. 정신의학적 교육을 받은 가족과 환자들이 치료에 더 욱 협조적이고 치료에 대한 반응이 더 좋다. 정신의학적 교육 은 병에 대한 정보와 다양한 치료적 접근에 대한 것들을 포함 하고 있어야 한다.

권고사항 2. $\mathrm{ADHD}$ 환자에게는 약물치료 외에 효과가 검증 된 부모교육 및 인지행동치료, 특수교육적 행동치료를 병행할 것을 추천한다.

권고사항 $3 . \mathrm{ADHD}$ 의 주증상인 주의력결핍, 과잉행동, 충동 성 문제의 정도가 경할 경우, 6 세 미만일 경우는 일차적으로 비약물치료를 고려할 것을 추천한다.

권고사항 4. 불안장애, 우울장애, 대인관계 문제 등이 동반되 어 있는 경우에는 약물치료 외 비약물치료 병행을 추천한다.

중심 단어:주의력결핍 과잉행동장애; 비약물치료; 치료 권고안.

\section{Conflicts of Interest}

The authors have no financial conflicts of interest.

\section{REFERENCES}

1) Subcommittee on Attention-Deficit/Hyperactivity Disorder; Steering Committee on Quality Improvement and Management, Wol- 
raich M, Brown L, Brown RT, DuPaul G, et al. ADHD: clinical practice guideline for the diagnosis, evaluation, and treatment of attentiondeficit/hyperactivity disorder in children and adolescents. Pediatrics 2011;128:1007-1022.

2) Atkinson M, Hollis C. NICE guideline: attention deficit hyperactivity disorder. Arch Dis Child Educ Pract Ed 2010;95:24-27.

3) Bai GN, Wang YF, Yang L, Niu WY. Effectiveness of a focused, brief psychoeducation program for parents of ADHD children: improvement of medication adherence and symptoms. Neuropsychiatr Dis Treat 2015;11:2721-2735

4) Ferrin M, Moreno-Granados JM, Salcedo-Marin MD, Ruiz-Veguilla M, Perez-Ayala V, Taylor E. Evaluation of a psychoeducation programme for parents of children and adolescents with ADHD: immediate and long-term effects using a blind randomized controlled trial. Eur Child Adolesc Psychiatry 2014;23:637-647.

5) Ferrin M, Perez-Ayala V, El-Abd S, Lax-Pericall T, Jacobs B, Bilbow A, et al. A randomized controlled trial evaluating the efficacy of a psychoeducation program for families of children and adolescents with ADHD in the United Kingdom: results after a 6-month followup. J Atten Disord 2016 Feb 2 [Epub ahead of print]. http://dx.doi. org/10.1177/1087054715626509.

6) Montoya A, Colom F, Ferrin M. Is psychoeducation for parents and teachers of children and adolescents with ADHD efficacious? A systematic literature review. Eur Psychiatry 2011;26:166-175.

7) Martin A, Volkmar FR. Lewis's child and adolescent psychiatry: a comprehensive textbook. 4th ed. Philadelphia: Lippincott Williams \& Wilkins;2007.

8) Vidal R, Bosch R, Nogueira M, Gómez-Barros N, Valero S, Palomar G, et al. Psychoeducation for adults with attention deficit hyperactivity disorder vs. cognitive behavioral group therapy: a randomized controlled pilot study. J Nerv Ment Dis 2013;201:894-900.

9) Hirvikoski T, Waaler E, Lindström T, Bölte S, Jokinen J. Cognitive behavior therapy-based psychoeducational groups for adults with ADHD and their significant others (PEGASUS): an open clinical feasibility trial. Atten Defic Hyperact Disord 2015;7:89-99.

10) Kazdin AE. Parent management training: evidence, outcomes, and issues. J Am Acad Child Adolesc Psychiatry 1997;36:1349-1356.

11) Mabe PA, Turner MK, Josephson AM. Parent management training. Child Adolesc Psychiatr Clin N Am 2001;10:451-464

12) Michelson D, Davenport C, Dretzke J, Barlow J, Day C. Do evidencebased interventions work when tested in the "real world?" A systematic review and meta-analysis of parent management training for the treatment of child disruptive behavior. Clin Child Fam Psychol Rev 2013;16:18-34.

13) Rimestad ML, Lambek R, Zacher Christiansen H, Hougaard E. Short- and long-term effects of parent training for preschool children with or at risk of ADHD: a systematic review and meta-analysis. J Atten Disord 2016 May 14 [Epub ahead of print]. http://dx.doi.org/ $10.1177 / 1087054716648775$.

14) Furlong M, McGilloway S, Bywater T, Hutchings J, Smith SM, Donnelly M. Cochrane review: behavioural and cognitive-behavioural group-based parenting programmes for early-onset conduct problems in children aged 3 to 12 years (review). Evid Based Child Health 2013;8:318-692.

15) Colalillo S, Johnston C. Parenting cognition and affective outcomes following parent management training: a systematic review. Clin Child Fam Psychol Rev 2016;19:216-235.

16) Kazdin AE. Parent management training: treatment for oppositional, aggressive, and antisocial behavior in children and adolescents. 1st ed. New York: Oxford University Press;2008.

17) Danforth JS. A flow chart of behavior management strategies for families of children with co-occurring attention-deficit hyperactivity disorder and conduct problem behavior. Behav Anal Pract 2016;9: 64-76.

18) Babinski DE, Waxmonsky JG, Waschbusch DA, Pelham WE Jr. Be- havioral observations of parents with ADHD during parent training. J Atten Disord 2015 Apr 13 [Epub ahead of print]. http://dx.doi.org/ $10.1177 / 1087054715580843$.

19) Jans T, Jacob C, Warnke A, Zwanzger U, Groß-Lesch S, Matthies S, et al. Does intensive multimodal treatment for maternal ADHD improve the efficacy of parent training for children with ADHD? A randomized controlled multicenter trial. J Child Psychol Psychiatry 2015; $56: 1298-1313$

20) Chronis-Tuscano A, Wang CH, Strickland J, Almirall D, Stein MA. Personalized treatment of mothers with ADHD and their young atrisk children: a SMART pilot. J Clin Child Adolesc Psychol 2016;45: 510-521.

21) Maliken AC, Katz LF. Exploring the impact of parental psychopathology and emotion regulation on evidence-based parenting interventions: a transdiagnostic approach to improving treatment effectiveness. Clin Child Fam Psychol Rev 2013;16:173-186.

22) Steeger CM, Gondoli DM, Gibson BS, Morrissey RA. Combined cognitive and parent training interventions for adolescents with ADHD and their mothers: a randomized controlled trial. Child Neuropsychol 2016;22:394-419.

23) Hosainzadeh Maleki Z, Mashhadi A, Soltanifar A, Moharreri F, Ghanaei Ghamanabad A. Barkley's parent training program, working memory training and their combination for children with ADHD: attention deficit hyperactivity disorder. Iran J Psychiatry 2014;9:4754.

24) Huang YH, Chung CY, Ou HY, Tzang RF, Huang KY, Liu HC, et al. Treatment effects of combining social skill training and parent training in Taiwanese children with attention deficit hyperactivity disorder. J Formos Med Assoc 2015;114:260-267.

25) Pianta RC. Enhancing relationships between children and teachers. Washington, DC: American Psychological Association;1999.

26) Pfiffner LJ, DuPaul GJ. Treatment of ADHD in school settings. In: Barkley RA, editor. Attention deficit hyperactivity disorder: a handbook for diagnosis and treatment. New York: Guilford Press;1998. p.458-490.

27) McMahon RJ, Forehand RL. Helping the noncompliant child: family-based treatment for oppositional behavior. New York: Guilford Press;2003.

28) Pfiffner LJ. All about adhd: the complete practical guide for classroom teachers (teaching strategies). New York: Scholastic Prof Book Div; 1996.

29) Pfiffner LJ, Rosén LA, O'Leary SG. The efficacy of an all-positive approach to classroom management. J Appl Behav Anal 1985;18:257261.

30) Weiss M, Murray C, Wasdell M, Greenfield B, Giles L, Hechtman L. A randomized controlled trial of CBT therapy for adults with ADHD with and without medication. BMC Psychiatry 2012;12:30.

31) Power TJ, Mautone JA. Best practices in linking families and schools to educate children with attention problems. In: Thomas A, Grimes $\mathrm{J}$, editors. Best practices in school psychology. Bethesda: National Association of School Psychologists;2008. p.839-851.

32) Power TJ, Hess LE, Bennett DS. The acceptability of interventions for attention-deficit hyperactivity disorder among elementary and middle school teachers. J Dev Behav Pediatr 1995;16:238-243.

33) Shapiro ES. Academic skills problems: direct assessment and intervention. 4th ed. New York: Guilford Press;2011.

34) Zentall SS. Research on the educational implications of attention deficit hyperactivity disorder. Except Child 1993;60:143-153.

35) Power TJ, Karustis JL, Habboushe DF. Homework success for children with ADHD: a family-school intervention program. New York: Guilford Press;2001.

36) DuPaul GJ, Ervin RA, Hook CL, McGoey KE. Peer tutoring for children with attention deficit hyperactivity disorder: effects on classroom behavior and academic performance. J Appl Behav Anal 1998; 31:579-592. 
37) Hook CL, DuPaul GJ. Parent tutoring for students with attentiondeficit/hyperactivity disorder: effects on reading performance at home and school. School Psych Rev 1999;28:60-75.

38) DuPaul GJ, Stoner G. ADHD in the schools: assessment and intervention strategies. New York: Guilford Press;2014.

39) Hoza B, Mrug S, Gerdes AC, Hinshaw SP, Bukowski WM, Gold JA, et al. What aspects of peer relationships are impaired in children with attention-deficit/hyperactivity disorder? J Consult Clin Psychol 2005; 73:411-423.

40) Hoza B. Peer functioning in children with ADHD. J Pediatr Psychol 2007;32:655-663

41) Pfiffner LJ, McBurnett K. Social skills training with parent generalization: treatment effects for children with attention deficit disorder J Consult Clin Psychol 1997;65:749-757.

42) Hoza B, Mrug S, Pelham WE Jr, Greiner AR, Gnagy EM. A friendship intervention for children with attention-deficit/hyperactivity disorder: preliminary findings. J Atten Disord 2003;6:87-98.

43) Fabiano GA, Pelham WE Jr, Karmazin K, Kreher J, Panahon CJ, Carlson C. A group contingency program to improve the behavior of elementary school students in a cafeteria. Behav Modif 2008;32: 121-132.

44) Leff SS, Costigan TE, Power TJ. Using participatory-action research to develop a playground-based prevention program. J School Psychol 2004;42:3-21.

45) Antshel KM, Faraone SV, Gordon M. Cognitive behavioral treatment outcomes in adolescent ADHD. J Atten Disord 2014;18:483-495.

46) Emilsson B, Gudjonsson G, Sigurdsson JF, Baldursson G, Einarsson E, Olafsdottir H, et al. Cognitive behaviour therapy in medication-treated adults with ADHD and persistent symptoms: a randomized controlled trial. BMC Psychiatry 2011;11:116.

47) Durlak JA, Fuhrman T, Lampman C. Effectiveness of cognitive-behavior therapy for maladapting children: a meta-analysis. Psychol Bull 1991;110:204-214.

48) Froelich J, Doepfner M, Lehmkuhl G. Effects of combined cognitive behavioural treatment with parent management training in ADHD. Behav Cogn Psychother 2002;30:111-115.

49) Battagliese G, Caccetta M, Luppino OI, Baglioni C, Cardi V, Mancini F, et al. Cognitive-behavioral therapy for externalizing disorders: a meta-analysis of treatment effectiveness. Behav Res Ther 2015;75:60-71.

50) Abikoff H, Gittelman R. Hyperactive children treated with stimulants. Is cognitive training a useful adjunct? Arch Gen Psychiatry 1985;42:953-961

51) Storebø OJ, Skoog M, Damm D, Thomsen PH, Simonsen E, Gluud C. Social skills training for attention deficit hyperactivity disorder (ADHD) in children aged 5 to 18 years. Cochrane Database Syst Rev 2011;(12):CD008223.

52) Gresham FM, Elliot SN. Social skills rating system manual. Circle Pines: American Guidance Service; 1990.

53) Lane KL, Givner CC, Pierson MR. Teacher expectations of student behavior: social skills necessary for success in elementary school classrooms. J Spec Educ 2004;38:104-110.

54) Bracken SS, Fischel JE. Relationships between social skills, behavioral problems, and school readiness for head start children. NHSA Dialog 2007;10:109-126.

55) Gresham FM, Elliott SN. Social skills intervention guide: systematic approaches to social skills training. Spec Serv Sch 1993;8:137-158.

56) Rockhill CM, Vander Stoep A, McCauley E, Katon WJ. Social competence and social support as mediators between comorbid depressive and conduct problems and functional outcomes in middle school children. J Adolesc 2009;32:535-553.

57) Durlak JA, Weissberg RP. The impact of after-school programs that promote personal and social skills. Chicago: Collaborative for Academic, Social, and Emotional Learning (CASEL);2007.

58) Fohlmann AH. Social færdighedstræning [Social skills training].
In: Kjær SK, Nordentoft M, Melau M, Iversen T, editors. Psykose hos unge. Symptomer, behandling og fremtid [Psychosis in the young. Symptoms, treatment and the future]. Copenhagen: Psykiatrifondens Forlag;2009. p.161-189.

59) Martin T. Social skills training. In: Liberman RP, editor. Psychiatric rehabilitation of chronic mental patients. Washington, DC: American Psychiatric Press;1988. p.147-198.

60) Spence SH. Social skills training with children and young people: theory, evidence and practice. Child Adolesc Ment Health 2003;8: 84-96.

61) Barkley RA. Adolescents with attention-deficit/hyperactivity disorder: an overview of empirically based treatments. J Psychiatr Pract 2004;10:39-56.

62) de Boo GM, Prins PJ. Social incompetence in children with ADHD: possible moderators and mediators in social-skills training. Clin Psychol Rev 2007;27:78-97.

63) Gol D, Jarus T. Effect of a social skills training group on everyday activities of children with attention-deficit-hyperactivity disorder. Dev Med Child Neurol 2005;47:539-545.

64) Cordier R, Munro N, Wilkes-Gillan S, Docking K. The pragmatic language abilities of children with ADHD following a play-based intervention involving peer-to-peer interactions. Int J Speech Lang Pathol 2013;15:416-428.

65) Gooding LF. The effect of a music therapy social skills training program on improving social competence in children and adolescents with social skills deficits. J Music Ther 2011;48:440-462.

66) Wagner SM, McNeil CB. Parent-child interaction therapy for ADHD: a conceptual overview and critical literature review. Child Fam Behav Ther 2008;30:231-256.

67) Landreth GL. Play therapy: the art of the relationship. 3rd ed. New York: Routledge;2012.

68) Thomas R, Zimmer-Gembeck MJ. Behavioral outcomes of parentchild interaction therapy and triple P-positive parenting program: a review and meta-analysis. J Abnorm Child Psychol 2007;35:475-495.

69) Pliszka S; AACAP Work Group on Quality Issues. Practice parameter for the assessment and treatment of children and adolescents with attention-deficit/hyperactivity disorder. J Am Acad Child Adolesc Psychiatry 2007;46:894-921.

70) Wilkes-Gillan S, Bundy A, Cordier R, Lincoln M, Chen YW. A randomised controlled trial of a play-based intervention to improve the social play skills of children with attention deficit hyperactivity disorder (ADHD). PLoS One 2016;11:e0160558.

71) Bratton SC, Ray D, Rhine T, Jones L. The efficacy of play therapy with children: a meta-analytic review of treatment outcomes. Prof Psychol Res Pr 2005;36:376-390.

72) Choi JA. Literature review of play therapy intervention for children with ADHD. Fam Environ Res 2012;50:125-138.

73) Ray DC, Schottelkorb A, Tsai MH. Play therapy with children exhibiting symptoms of attention deficit hyperactivity disorder. Int $\mathbf{J}$ Play Ther 2007;16:95-111.

74) Sprich SE, Safren SA, Finkelstein D, Remmert JE, Hammerness P. A randomized controlled trial of cognitive behavioral therapy for ADHD in medication-treated adolescents. J Child Psychol Psychiatry 2016;57:1218-1226.

75) Jensen CM, Amdisen BL, Jørgensen KJ, Arnfred SM. Cognitive behavioural therapy for ADHD in adults: systematic review and metaanalyses. Atten Defic Hyperact Disord 2016;8:3-11.

76) Mongia M, Hechtman L. Cognitive behavior therapy for adults with attention-deficit/hyperactivity disorder: a review of recent randomized controlled trials. Curr Psychiatry Rep 2012;14:561-567.

77) Young Z, Moghaddam N, Tickle A. The efficacy of cognitive behavioral therapy for adults with ADHD: a systematic review and metaanalysis of randomized controlled trials. J Atten Disord 2016 Aug 22 [Epub ahead of print]. http://dx.doi.org/10.1177/1087054716664413.

78) Dittner AJ, Rimes KA, Russell AJ, Chalder T. Protocol for a proof of 
concept randomized controlled trial of cognitive-behavioural therapy for adult ADHD as a supplement to treatment as usual, compared with treatment as usual alone. BMC Psychiatry 2014;14:248.

79) Lipton MA, Mayo JP. Diet and hyperkinesis--an update. J Am Diet Assoc 1983;83:132-134.

80) Kanarek RB. Does sucrose or aspartame cause hyperactivity in children? Nutr Rev 1994;52:173-175.

81) Akhondzadeh S, Mohammadi MR, Khademi M. Zinc sulfate as an adjunct to methylphenidate for the treatment of attention deficit hyperactivity disorder in children: a double blind and randomized trial [ISRCTN64132371]. BMC Psychiatry 2004;4:9.

82) Konofal E, Lecendreux M, Deron J, Marchand M, Cortese S, Zaïm M, et al. Effects of iron supplementation on attention deficit hyperactivity disorder in children. Pediatr Neurol 2008;38:20-26.

83) Raz R, Gabis L. Essential fatty acids and attention-deficit-hyperactivity disorder: a systematic review. Dev Med Child Neurol 2009;51: 580-592.

84) Johnson M, Ostlund S, Fransson G, Kadesjö B, Gillberg C. Omega-3/ omega- 6 fatty acids for attention deficit hyperactivity disorder: a randomized placebo-controlled trial in children and adolescents. J Atten Disord 2009;12:394-401.

85) Lubar JF, Shouse MN. EEG and behavioral changes in a hyperkinetic child concurrent with training of the sensorimotor rhythm (SMR): a preliminary report. Biofeedback Self Regul 1976;1:293-306.

86) Arns M, de Ridder S, Strehl U, Breteler M, Coenen A. Efficacy of neurofeedback treatment in ADHD: the effects on inattention, impulsivity and hyperactivity: a meta-analysis. Clin EEG Neurosci 2009;40:180-189.

87) Gevensleben H, Holl B, Albrecht B, Schlamp D, Kratz O, Studer P, et al. Distinct EEG effects related to neurofeedback training in children with ADHD: a randomized controlled trial. Int J Psychophysiol 2009;74:149-157.

88) Gevensleben H, Holl B, Albrecht B, Vogel C, Schlamp D, Kratz O, et al. Is neurofeedback an efficacious treatment for ADHD? A randomised controlled clinical trial. J Child Psychol Psychiatry 2009; 50:780-789.

89) Duric NS, Assmus J, Gundersen D, Elgen IB. Neurofeedback for the treatment of children and adolescents with ADHD: a randomized and controlled clinical trial using parental reports. BMC Psychi- atry 2012;12:107.

90) Monastra VJ, Monastra DM, George S. The effects of stimulant therapy, EEG biofeedback, and parenting style on the primary symptoms of attention-deficit/hyperactivity disorder. Appl Psychophysiol Biofeedback 2002;27:231-249.

91) Fuchs T, Birbaumer N, Lutzenberger W, Gruzelier JH, Kaiser J. Neurofeedback treatment for attention-deficit/hyperactivity disorder in children: a comparison with methylphenidate. Appl Psychophysiol Biofeedback 2003;28:1-12.

92) Rossiter T. The effectiveness of neurofeedback and stimulant drugs in treating AD/HD: part II. Replication. Appl Psychophysiol Biofeedback 2004;29:233-243.

93) Sonuga-Barke EJ, Brandeis D, Cortese S, Daley D, Ferrin M, Holtmann M, et al. Nonpharmacological interventions for ADHD: systematic review and meta-analyses of randomized controlled trials of dietary and psychological treatments. Am J Psychiatry 2013;170: 275-289.

94) Cortese S, Ferrin M, Brandeis D, Holtmann M, Aggensteiner P, Daley $D$, et al. Neurofeedback for attention-deficit/hyperactivity disorder: meta-analysis of clinical and neuropsychological outcomes from randomized controlled trials. J Am Acad Child Adolesc Psychiatry 2016;55:444-455.

95) Klingberg T, Fernell E, Olesen PJ, Johnson M, Gustafsson P, Dahlström K, et al. Computerized training of working memory in children with ADHD--a randomized, controlled trial. J Am Acad Child Adolesc Psychiatry 2005;44:177-186.

96) Beck SJ, Hanson CA, Puffenberger SS, Benninger KL, Benninger WB. A controlled trial of working memory training for children and adolescents with ADHD. J Clin Child Adolesc Psychol 2010;39:825836.

97) Chacko A, Feirsen N, Bedard AC, Marks D, Uderman JZ, Chimiklis A. Cogmed working memory training for youth with ADHD: a closer examination of efficacy utilizing evidence-based criteria. J Clin Child Adolesc Psychol 2013;42:769-783.

98) Chacko A, Bedard AC, Marks DJ, Feirsen N, Uderman JZ, Chimiklis A, et al. A randomized clinical trial of cogmed working memory training in school-age children with ADHD: a replication in a diverse sample using a control condition. J Child Psychol Psychiatry 2014;55:247-255. 